\title{
ACOUSTIC SCORES AND SYMBOLIC MISMATCH PENALTIES IN PHONE LATTICES
}

\author{
Louis ten Bosch, Annika Hämäläinen, Odette Scharenborg, Lou Boves \\ CLST, Radboud University Nijmegen, Nijmegen, The Netherlands \\ \{L.tenBosch, A.Hamalainen, O.Scharenborg, L.Boves\}@let.ru.nl
}

\begin{abstract}
This paper builds on previous work that aims at unraveling the structure of the speech signal by means of using probabilistic representations. The context of this work is a multi-pass speech recognition system in which a phone lattice is created and used as a basis for a lexical search in which symbolic mismatches are allowed at certain costs. The focus is on the optimization of the costs of phone insertions, deletions and substitutions that are used in the lexical decoding pass. Two optimization approaches are presented, one related to a multi-pass computational model for human speech recognition, the other based on a decoding in which Bayes' risks are minimized. In the final section, the advantages of these optimization methods are discussed and compared.
\end{abstract}

\section{INTRODUCTION}

Currently, there is a growing interest in revisiting multi-pass approaches for automatic speech recognition (ASR) [2] [6] [12]. In a multi-pass system, often a (weighted) phone lattice is created in the first pass, followed by a lexical search, in which additional specialized decoding steps can be applied or more detailed information, e.g., morphological and domain knowledge, can be used. Compared with an integrated search, there are at least two advantages to such an approach. First, a multi-pass approach is useful when spoken keywords are to be detected from a potentially wide range of domains such as meetings, interviews, voice mails, and lectures (cf. [16]): these approaches all share the same phonedecoding pass. A second advantage is the greater flexibility with which specialized knowledge sources can be brought to bear in subsequent passes, such as cross-word triphones, phonotactic restrictions, morphology, long-span syntax, etc. (e.g., [2]).

Weighted phone lattices have shown to be very versatile in a recently developed computational model for human word processing (SpeM). SpeM is a multi-pass decoder in which a phone recognizer in the first pass generates a phone lattice that is used in the subsequent lexical search module. SpeM has been used to successfully model a number of key results from psycholinguistic experiments [9] [10]. In SpeM, mismatches between phone sequences in the lattice and the phone representations according to the lexicon are dealt with in a more flexible manner than in previous computational models of human auditory word recognition. However, although SpeM does offer more flexibility, in all experiments conducted so far the same penalty was applied for each substitution in the lexical decoding, (and mutatis mutandis also for insertions and deletions. Thus, only three indiscriminate penalties have been used.
In SpeM, the acoustic scores (costs) in the phone lattice computed by the phone recognizer, and the penalties of phone mismatches (also called 'symbolic mismatches') interact in a complex way. For example, if the mismatch cost is low, the likelihood of associating phone paths in the lattice to a word sequence will be large (since mismatches are cheap), and therefore the probability of decoding the correct word sequence might suffer. On the other hand, if the mismatch penalties are high, phone paths must be canonical themselves (and are therefore less likely to have a low cost) to induce a lexical solution - which evidently decreases the likelihood of finding any lexical solution in the phone graph. Therefore, the correct trade-off between values of 'symbolic' mismatches on the one hand and the acoustic costs in the phone lattice on the other hand is essential for the success of any lexical search pass that takes the phone lattice as input.

This paper focuses on approaches to find an optimal balance between acoustic scores and symbolic mismatch penalties. More specifically, our aim is to investigate how the costs for insertions, deletions, and substitutions affect the likelihood of finding the phone sequence that corresponds to the correct word sequence (as defined by the annotation on word level), and how these optimal costs relate to the acoustic costs in the phone lattice. To that end, we discuss two related optimization approaches. In Section 2, we will deal with the optimization in the context of the SpeM decoding, in which the optimal values for mismatch penalties have been found by a systematic search based on insight in the structure of the search space. In Section 3 we will deal with another, datadriven way to derive optimal mismatch penalties (Minimal Bayesian Risk Decoding). In the final section, we relate both approaches and discuss their advantages and disadvantages.

\section{LEXICAL PHONE PATHS AND SYMBOLIC MISMATCHES}

Our starting point is a phone lattice generated by a free phone loop, guided by a phone bigram. In general, it is often the case that the canonical phone transcription of a word (sequence) is not present in the phone graph, even though a phone lattice may consist of millions of phone paths. For instance, earlier research showed that for $34.9 \%$ of a set of 885 phone lattices that were created with a phone bigram, the canonical phone transcription of the utterance was not present in the phone graph [13]. Therefore, phone insertions, deletions, and substitutions must be dealt with to decode utterances in terms of lexical tokens.

In this section, we investigate under which conditions correct 'lexical' phone paths can be found in phone lattices if symbolic mismatches are allowed. A phone path is 'lexical' if it is a series of 
phone sequences corresponding to words from the lexicon, and 'correct' if it is made up of those phone sequences that correspond to the word transcription.

The approach consisted of the following steps.

1) For each utterance, a phone lattice is created using acoustic models trained on an independent training set.

2) A word search algorithm is used to search phone paths associated with sequences of words - allowing symbolic mismatches (phone insertions, deletions, and substitutions) at a specific cost.

3) Symbolic mismatch penalties are chosen to optimize the likelihood of the correct lexical phone path being the best among all other lexical phone paths through the lattice.

Below, these steps are described in more detail.

\subsection{Data and feature extraction}

We used a sub-corpus of the Spoken Dutch Corpus, a 9-millionword database comprising 1000 hours of speech annotated on various tiers (e.g. orthographic, prosodic, part-of-speech) [7]. This sub-corpus contains read speech from a Dutch spoken library for the blind. The material comprises word labels as well as manually verified word-level segmentations.

The data in the sub-corpus were divided into three sets: a training set, test set and a development test set (3500, 687 and 610 sentences, respectively). Table I gives an overview of the number of word tokens, speakers, and the amount of speech material per set.

Table I. Dataset used in this study.

\begin{tabular}{|l|c|c|c|c|}
\hline & Training & Test & Development & Total \\
\hline \hline $\begin{array}{l}\text { Orthographic } \\
\text { word tokens }\end{array}$ & 45,172 & 7,917 & 7,507 & 60,596 \\
\hline $\begin{array}{l}\text { Speakers/ } \\
\text { Female/Male }\end{array}$ & $125 /$ & $125 /$ & $125 /$ & $125 /$ \\
\hline $\begin{array}{l}\text { Duration } \\
\text { (hh:mm:ss) }\end{array}$ & $04: 51: 27$ & $00: 51: 34$ & $00: 48: 13$ & $06: 31: 14$ \\
\hline
\end{tabular}

Feature extraction was carried out at a frame rate of $10 \mathrm{~ms}$ using a 25-ms Hamming window. A pre-emphasis factor of 0.97 was employed. 12 Mel Frequency Cepstral Coefficients (MFCCs) and log-energy with corresponding first and second order time derivatives were used. Channel normalization was applied by means of CMN over complete recordings (with a mean duration of 3.5 minutes). For training and testing purposes, the data were chunked to short utterances without long utterance-internal pauses. The feature extraction was performed using HTK [15].

The training corpus was used to create 39 context-independent acoustic models (3-state left-to-right HMMs with 8 Gaussians per state) on the basis of the lexical phone transcriptions. The lexicon covered all words in training, test, and development set, and contained one pronunciation variant per word.

A phone bigram ('phonotactic model') has been trained on the lexical phone transcriptions of the training corpus. Since leading and trailing silences as well as inter-word silences are annotated on the word level, this method automatically includes bigrams of the form $P($ sill $\phi)$ and $P(\phi \mid s i l)$ ( $\phi$ denoting an arbitrary phone).

\subsection{Phone lattice parameter settings}

For the construction of the phone lattices, the phone insertion probability and phone-LM factor were tuned using the development set such that the number of phones of the first-best phone path and the number of phones of the canonical phone transcription were equal on average. This was done since mismatches with respect to these lengths will bias the values for phone insertions and deletions. Furthermore, the LM factor was chosen to be as close as possible to 0 (i.e., the decoding is as unbiased as possible). As a result, the insertion log probability and the LM-factor were set to -6 and 4 , respectively. The beam width has been chosen large enough to make sure that the time-averaged number of arcs with different phone labels is close to 3, i.e., a plausible number of realistic phonetic alternatives is present in the lattice. The resulting lattices have on average 810 arcs/second, with 12-18 arcs alive per time slice of $10 \mathrm{~ms}$, and the phone paths contain on average 12.8 phones/second.

\subsection{The lexical decoder and the search space}

The search for the lexical phone paths through the phone lattices was based on an FST decoder. This decoder is constructed by interfacing an HTK phone decoder with the AT\&T wFST software [5]. The decoding is implemented by a finite state composition of the phone lattice and an FST. This FST is based on the lexical tree, but expanded by including additional arcs with appropriate costs: arcs that accept any input and write out the null label $\varepsilon$ (modeling phone insertions), arcs that accept $\varepsilon$ and write out a phone label (deletions), and arcs that accept a phone $\phi 2$ and write out $\phi 1$ (substitutions). All insertions share the same penalty value (idem for deletions and substitutions, resulting in three penalties).

In [13] ten Bosch et al. show that the penalties for symbolic mismatches are to be chosen within certain bounds related to the acoustic scores in the original phone lattice. The structure of the eventual search space is a union of lattices, such that each of these lattices is associated with exactly one triplet $[I, D, S]$ of nonnegative integers $I, D$ and $S$ (representing the number of insertions, deletions and substitutions, respectively, in that particular lattice). Together the parameters $I, D$ and $S$ determine the cost that must be added to the original acoustic cost distribution. The problem of finding optimal symbolic costs is greatly reduced by restricting the parameters to those regions in the cost space that avoid these distributions to become disjoint.

\subsection{Decoding accuracy}

Now we investigate how the costs for insertions, deletions, and substitutions affect the likelihood of finding the phone sequence that corresponds to the correct word sequence (as defined by the annotation on word level), and how these optimal costs relate to the acoustic costs in the phone lattice. The decoding accuracy is defined as the proportion of phone lattices with the property that, after composition with the lexical FST, the correct lexical phone sequence is the cheapest among all lexical phone sequences. This property will guarantee that the lexical search will be able to correctly recognize all words in the entire utterance.

This search is actually three-dimensional, but the contour plot in Figure 1 shows an example of the behavior of the decoding accuracy as a function of the insertion penalty (along the x-axis) and the substitution penalty (along the $y$-axis). In the figure, the deletion penalty is constant (2.5). 
The performance of 0.68 (68 percent of the lattices had the correct word sequence corresponding to the cheapest path) was obtained for a substitution, insertion and deletion penalty of about $3.5,2.4$, and 2.5 , respectively. Thus for $68 \%$ of the graphs, the correct lexical phone sequence was the cheapest among all lexical phone sequences. For these 'cheapest sequences', the proportion of symbolic mismatches compared to the path length depends on the utterance and varies from 18 to 41 percent. For comparison: using a similar technique, [1] reports an average of 26.6 percent phone mismatches on phonetically labeled manually transcribed spontaneous speech. The performance difference can at least in part, be explained by the fact that we only searched for canonical paths in the lattice, whereas it is evident that the actual pronunciation often deviated from the canonical. This would result in a minimum mismatch rate of about $10 \%$ for read speech if the phone recognizer would detect the 'exact' sequence of phones in a careful manual transcription.

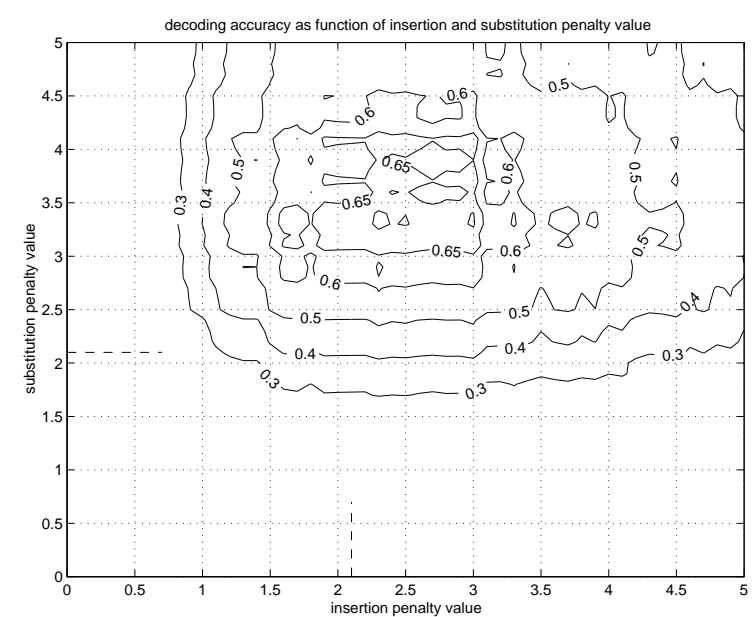

Figure 1. Decoding accuracy as a function of the insertion penalty (along the $x$-axis) and the substitution penalty (along the y-axis). The horizontal and vertical dashed lines indicate the average acoustic 'penalty', i.e. the difference between acoustic score of competing phones.

The optimal symbolic penalties for insertions and deletions are a factor 1.2 larger than the acoustic mismatch costs, while the substitution penalty is 1.4 to 1.5 times larger than the insertion and deletion penalties. We observe that these ratios are independent of the acoustic costs: since all numerical operations for the total cost along paths are linear, the symbolic penalties scale with the acoustic costs and thus the ratios are constant.

\section{DATA-DERIVED PHONE-PHONE SUBSTITUTION COSTS}

\subsection{Minimum Bayesian Risk decoding}

In the previous section, the optimal mismatch costs were found by systematic search that was motivated by the structure of the distribution of path costs. One of the evident drawbacks of such a method is the fact that all substitutions are penalized by the same amount, independent of the phonetic or acoustic distance between source and target phone. However, the use of much more parameters becomes prohibitive.

Expression (1) provides the mathematical formulation of the optimization of the probability to find the correct phone path in the phone lattice according to the SpeM decoding framework. The signal $X$ is given, $P$ is the hypothesized lexical path, and $Q$ is a path variable, running over the set of all paths available in the phone lattice. The term $-\log (\mathrm{P}(X \mid Q))$ denotes the acoustic score in the phone lattice, while $d(P, Q)$ denotes the sum of all penalties for symbolic mismatches between the phone sequences $P$ and $Q$.

$$
P_{c}=\operatorname{argmin}_{P}\left\{\min _{Q}(-\log (P(X \mid Q)+d(P, Q))\}\right.
$$

Shafran \& Byrne [11] present a procedure for solving a similar decoding problem. Their approach is based on using a Minimum Bayes Risk (MBR) criterion given by Eq. 2:

$$
P_{c}=\arg \min _{P} \sum_{Q} C(P, Q) P(X \mid Q) P(Q)
$$

in which $X, Q$ and $P$ denote the signal, a path variable (running over all phone sequences in the lattice), and the resulting path, respectively. $C(P, Q)$ denotes the cost of rewriting the path $Q$ into the path $P$. Their aim is to automatically learn the substitution costs $C(P, Q)$ from data.

It can be observed that Eq. (1) and Eq. (2) have a similar structure: the $\min _{Q}$ is replaced by a sum, and $d(P, Q)$ can be interpreted as $C(P, Q)$ (both these terms are basically edit distances). Equation (1) aims at optimizing the probability of finding the correct lexical path in the lattice, while Equation (2) supports the search for optimal penalty values that are valid across all lexical paths in the lattice.

In order to contrast both approaches, we have defined an iterative scheme using Eq. (2) in order to estimate cost $C(P, Q)$ as follows:

(1) Decode the data using the current model (acoustic models and current parameter settings that define $C(P$, Q))

(2) Compute alignments between solution and reference transcription

(3) Compute the updated $C(P, Q)$ by setting $C(P, Q)$ equal to $-\log (\mathrm{P}(P \mid Q))$

Here, $C(P, Q)$ refers to the weighted edit distance, i.e. the minimum weighted number of modifications to be applied on the phone path $Q$ to obtain the phone path $P$. The scheme has been applied using the same speech data as in section 2. Furthermore, substitution costs have been trained for all combinations of five broad phonetic manner classes: plosive (stop), fricative, liquid, nasal, and (semi)vowel. The initial choice for bootstrapping these 25 substitution costs is given in Table IIa. This table is inspired by the optimal substitution penalty found by the SpeM decoding. Table IIb is the result after applying the iteration scheme on all phone lattices of the training set after the third iteration. The third iteration was chosen since from this iteration on, all matrix entries differ less than 1 percent compared to the values obtained after the third iteration. The evolution of the decoding accuracy ('Acc') after the first 5 iterations is presented in Table III. This shows that the Shafran-Byrne scheme is potentially able to outperform the SpeM search due to the feasibility of training more fine-grained 
mismatch costs - something that was not feasible with the SpeM approach.

Table II shows the penalty values in the same scale as used in the previous section. That means that they can be compared to 2.1 , the average acoustic cost for a symbolic mismatch.

\section{DISCUSSION AND CONCLUSION}

The balance between acoustic costs and symbolic mismatches is important for the performance of a multi-pass speech decoding system. In this paper, we discuss two approaches for finding the optimal balance, one in the context of the SpeM decoding and one based on Minimum Bayesian Risk (MBR) decoding. The underlying mathematical formulation of both methods is very similar. The advantage of the SpeM decoding is that the structure of the search space is known in terms of the three penalty values: the cost distribution is an overlay of smaller distributions that are spaced apart according to the symbolic mismatch values. This structure simplifies the optimisation, because it allows to restrict the search to specific sub-regions. We have shown that the MBR approach is able to train more refined categories of mismatch costs (by distinguishing more phone classes) methods.

TableI II (a, top). Initial substitution cost matrix. (b, bottom) Cost matrix after three iterations (see text).

\begin{tabular}{|l|l|l|l|l|l|}
\hline (a) & Plos & Fric & Liq & Nas & Vowel \\
\hline Plos & 0.0 & 3.5 & 3.5 & 3.5 & 3.5 \\
\hline Fric & 3.5 & 0.0 & 3.5 & 3.5 & 3.5 \\
\hline Liq & 3.5 & 3.5 & 0.0 & 3.5 & 3.5 \\
\hline Nas & 3.5 & 3.5 & 3.5 & 0.0 & 3.5 \\
\hline Vowel & 3.5 & 3.5 & 3.5 & 3.5 & 0.0 \\
\hline & & & & & \\
\hline (b) & Plos & Fric & Liq & Nas & Vowel \\
\hline Plos & 0.4 & 3.2 & 3.6 & 4.0 & 5.1 \\
\hline Fric & 3.0 & 0.6 & 3.5 & 3.6 & 3.9 \\
\hline Liq & 3.1 & 3.5 & 1.1 & 2.3 & 2.8 \\
\hline Nas & 3.6 & 3.9 & 2.2 & 0.9 & 3.2 \\
\hline Vowel & 5.2 & 3.9 & 2.6 & 3.4 & 1.4 \\
\hline
\end{tabular}

Table III Decoding accuracy in percent before the optimization (Iteration 0) and after a number of iteration steps (Iteration 1 to 5) using the MBR optimization scheme.

\begin{tabular}{|l|l|l|l|l|l|l|}
\hline Iteration & 0 & 1 & 2 & 3 & 4 & 5 \\
\hline Acc $(\%)$ & 68 & 71 & 71 & 72 & 72 & 72 \\
\hline
\end{tabular}

It is interesting to observe that the costs that are trained for withinbroad-phonetic-class substitutions by the MBR approach are larger than 0 . One might expect them to be identically zero. However, these positive values can be explained by the fact that these substitution costs also account for substations between non-equal plosives, non-equal nasals (e.g. $\mathrm{C}(/ \mathrm{p} /, / \mathrm{q} /), \mathrm{C}(/ \mathrm{m} /, / \mathrm{n} /)$ etc.). On average these costs are much smaller than any other costs between broad classes.

It is expected that further improvements can be obtained by allowing more fine-grained distinctions in the cost function. Focus of research in the near future will be the relation between the acoustic costs (perhaps computed on the basis of articulatory features [14]), symbolic mismatch penalties, the structure in phone lattices and the decoding of speech in terms of lexical tokens, applied on larger corpora and for spontaneous speech instead of read speech.

\section{REFERENCES}

[1] Bates, R., and Ostendorf, M. (2002). Modeling pronunciation variation in conversational speech using prosody. Workshop PMLA, Estes Park, USA.

[2] Demuynck, K., Laureys, T., van Compernolle, D., Van hamme, H. (2003). FLaVoR, a flexible architecture for LVCSR. Proceedings Eurospeech, Geneva, Switzerland. Pp. 1973-1976. http://www.esat.kuleuven.ac.be/ spch/projects/FLaVoR/

[3] Frankel, J., Wester, M., King, S. (2004). Articulatory feature recognition using dynamic Bayesian networks. ICSLP 2004, Korea (cdrom).

[4] King, S., and Taylor, P. (2000). Detection of phonological features in continuous speech using neural networks, Computer Speech and Language, 14(4), pp. 333-353.

[5] Mohri, M., Perreira, F., Riley, M (2003). AT\&T FSM Library $^{\mathrm{TM}}$, General-Purpose Finite-State Machine Software Tools.

[6] Ohtsuki, K., Hiroshima, N., Matsunaga, S., Hayashi, Y. (2004). Multi-pass ASR using vocabulary expansion. Proc. ICSLP, Jeju, South Korea, October 2004 (cdrom).

[7] Oostdijk, N. (2002). The design of the Spoken Dutch Corpus. In: Peters, P., Collins, P., Smith A. (Eds) New Frontiers of Corpus Research (pp. 105-112). Amsterdam: Rodopi.

[8] Ristad E.S. and Yianilos, P.N. (1998). A Surficial Pronunciation Model. Proc. ECSA Workshop on Modeling Pronunciation Variation for Automatic Speech Recognition, Rolduc, the Netherlands.

[9] Scharenborg, O., ten Bosch, L., Boves, L. (2003). 'Early Recognition' of Words in Continuous Speech Proc. ASRU workshop, St Thomas, US Virgin Islands (cdrom).

[10] Scharenborg, O., Norris, D., ten Bosch, L., McQueen, J.M., (accepted). "How should a speech recognizer work?". Will be published in Cognitive Science.

[11] Shafran I. and Byrne, W. (2004). Task-Specific Minimum Bayes-Risk Decoding using Learned Edit Distance. Interspeech 2004, vol. 3, pp. 1945-48, Jeju Islands, Korea, Oct. 4-8, 2004.

[12] Tang, M., Seneff, S., and Zue, V. (2003). Two-stage speech recognition using feature-based models: a preliminary study. Proc. ASRU workshop, St Thomas, US Virgin Islands (cdrom).

[13] Ten Bosch, L., Scharenborg, O. (2005). ASR Decoding in a Computational Model of Human Word Recognition. Proc. Interspeech 2005, Lisbon, Portugal (cdrom).

[14] Wester, M., Frankel, J., King, S. (2004). Asynchronous articulatory feature recognition using dynamic Bayesian networks. Workshop Beyond HMM, Kyoto, Japan.

[15] Young, S., Evermann, G., Hain, T., Kershaw, D., Moore, G., Odell, J., Ollason, D., Povey, D., Valtchev, V., and Woodland, P. (2002). The HTK Book (for HTK Version 3.2.1). Cambridge University Engineering Department, Cambridge, UK.

[16] Yu, P., and Seide, F. (2004). A hybrid-word/phoneme-based approach for improved vocabulary-independent search in spontaneous speech. Proc. Interspeech 2004, 293-296. 